\title{
Dupilumab for Atopic Dermatitis: The Silver Bullet We Have Been Searching for?
}

\author{
Ahsan Tameez Ud Din ${ }^{1}$, Ifrah Malik ${ }^{1}$, Daneyal Arshad ${ }^{1}$, Asim Tameez Ud Din ${ }^{1}$ \\ 1. Internal Medicine, Rawalpindi Medical University, Rawalpindi, PAK
}

Corresponding author: Ahsan Tameez Ud Din, ahsantameezuddinmalik@gmail.com

\begin{abstract}
Atopic dermatitis is a chronic inflammatory condition of the skin affecting a large number of people worldwide. Historically, this condition has been managed by topical corticosteroids and general skincare measures. The inadequacy of these management strategies has always driven efforts to find better drugs. Dupilumab has been recently approved for the management of atopic dermatitis. It is a human monoclonal antibody that inhibits the binding of key interleukins involved in the pathogenesis of atopic dermatitis, thus blocking the signaling mechanisms and disrupting the disease progression. Dupilumab reduces the severity and associated symptoms of atopic dermatitis. It improves the life quality of patients and reduces the anxiety associated with the disease. Combination therapy of dupilumab and topical corticosteroids is more effective than dupilumab monotherapy. The treatment-related adverse events include headache, injection site reaction, conjunctivitis, nasopharyngitis, and herpes viral infections. Moreover, the simultaneous use of live vaccines with dupilumab is contraindicated. It is also beneficial in the management of asthma, chronic rhinosinusitis, and eosinophilic esophagitis. In this review, we have discussed the clinical efficacy and safety profile of dupilumab in the management of atopic dermatitis.
\end{abstract}

Categories: Dermatology, Internal Medicine, Allergy/Immunology Keywords: atopic dermatitis, eczema, dupilumab, allergy

\section{Introduction And Background}

Atopic dermatitis is a chronic inflammatory condition of the skin characterized by pruritus and skin changes [1]. It affects $2-10 \%$ of adults worldwide and may be associated with other systemic disorders like asthma [2]. The underlying pathology of atopic dermatitis revolves mainly around type 2 immune-mediated reaction, with cytokines interleukin-4 (IL-4) and interleukin-13 (IL-13) playing a key role in its pathogenesis [3,4].

Received 03/31/2020

Review began 04/04/2020 Review ended 04/04/2020 Published 04/06/2020

\section{(c) Copyright 2020}

Tameez Ud Din et al. This is an open access article distributed under the terms of the Creative Commons Attribution License CC-BY 4.0., which permits unrestricted use, distribution, and reproduction in any medium, provided the original author and source are credited.
Owing to the chronic nature of the disease, the treatment of moderate-to-severe atopic dermatitis tends to be long term. Historically, topical corticosteroids and general skincare by emollients and non-irritative skin preparations have been the mainstay of management of atopic dermatitis $[1,5,6]$. The use of systemic agents (e.g., oral corticosteroids and immunosuppressants such as cyclosporine and methotrexate) and phototherapy are usually limited to the treatment of cases showing minimal response to the topical agents [7]. Long-term use of systemic agents is not recommended due to the risk of adverse side effects [5,7]. The inadequacy of the current mainstream treatment options has been the driving force behind the extensive efforts to find a safe and effective drug for the treatment of this ubiquitous skin disorder.

Dupilumab has been recently approved by the U.S. Food and Drug Administration (FDA) for the use in the treatment of atopic dermatitis. It is a human monoclonal antibody that targets the signaling mechanisms of cytokines IL-4 and IL-13 [8]. This review article aims to improve the understanding of this newly approved drug by comparing the findings of different clinical trials. The main objective of this study is to give a comprehensive view of the efficacy and safety profile of dupilumab.

\section{Review}

\section{Methods}

A literature search was performed using MeSH (Medical Subject Headings) terms and keywords on the PubMed database. A total of 20 articles were identified. The selected studies included clinical trials that demonstrated the efficacy, side effects, drug interactions, and other uses of dupilumab.

Two independent reviewers screened the abstracts and the full text of articles. After meticulous screening and a detailed discussion, eight studies were selected for the final review.

One author performed data extraction, whereas a second author analyzed the extracted data. The study variables that were analyzed included the name(s) of the author(s), year of study, design of the study, sample size, the severity of atopic dermatitis, efficacy, adjunctive therapy, safety, and drug interactions. 


\section{Mechanism of action}

Dupilumab is a human monoclonal antibody acting on the IL-4 receptor. Two types of IL-4 receptors present on a surface of many cells (e.g. B lymphocytes, eosinophils, dendritic cells, monocytes, macrophages, basophils, keratinocytes, etc.) are involved in the pathogenesis of type 2 allergic responses [9]. The binding of cytokine ligands to these receptors leads to a cascade of events that result in IgE (immunoglobulin E) class switching, TH2 (T-helper cell type 2) differentiation, and M2 macrophage polarization [10]. In atopic dermatitis, cytokines IL-4 and IL-13 play a key role in the modulation of the epidermal barrier and the stimulation of dermal inflammation and remodeling [11-13].

Evidence from a large number of clinical trials suggests that dupilumab acts by inhibiting the binding of IL-4 receptors to the main cytokines responsible for mediating the disease (IL-4 and IL-13). This results in the blockage of the signaling pathways, which disrupts the development and progression of allergic disorders [14].

\section{Route of administration and recommended dose}

Dupilumab is administered subcutaneously by injection. The patient may be taught the appropriate method of self-injection, which may reduce the inconvenience of repeated visits to a health care facility. The recommended dose of dupilumab is $300 \mathrm{mg}$ every two weeks after an initial loading dose of $600 \mathrm{mg}$ (two 300 $\mathrm{mg}$ injections at different sites) [15]. However, certain studies suggest that the dose of $300 \mathrm{mg}$ given weekly may be as or even more effective than the current recommended dosing schedule $[16,17]$.

\section{Clinical efficacy and quality of life}

The severity of atopic dermatitis and its effect on the quality of life is assessed by a variety of scoring systems that include subjective as well as objective measurements [18-22]. Table 1 lists the scoring systems frequently used by the investigators in the clinical trials concerning atopic dermatitis.

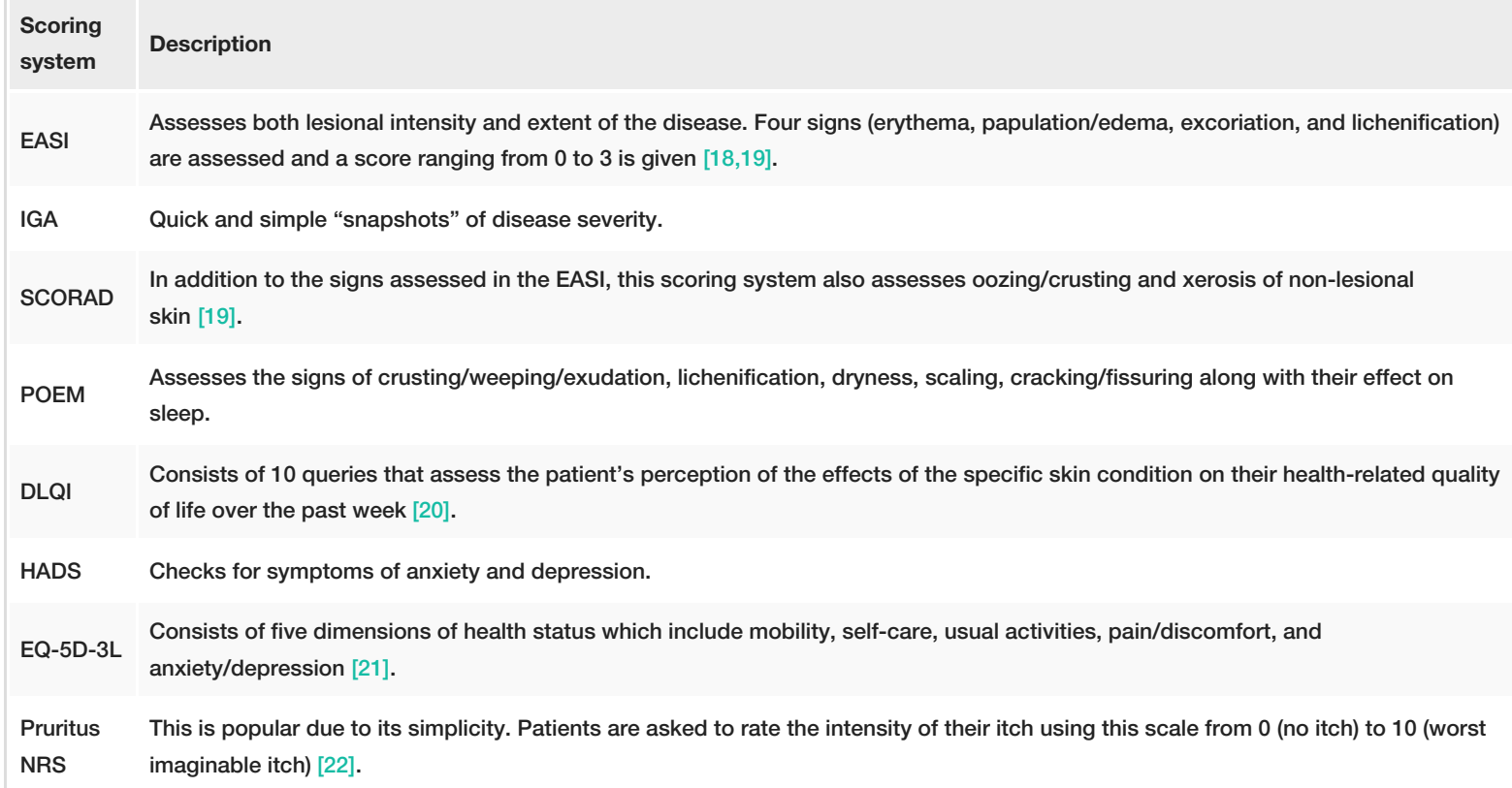

Pruritus This is popular due to its simplicity. Patients are asked to rate the intensity of their itch using this scale from 0 (no itch) to 10 (worst NRS imaginable itch) [22].

\section{TABLE 1: Scoring systems used in atopic dermatitis trials}

EASI, Eczema Area and Severity Index; IGA, Investigator Global Assessment; SCORAD, Scoring Atopic Dermatitis; POEM, Patient-Oriented Eczema Measure; DLQI, Dermatology Life Quality Index; HADS, Hospital Anxiety and Depression Scale; EQ-5D-3L, 5-dimension 3-level EuroQol; NRS,

Numeric Rating Scale

The clinical trials of dupilumab reported a reduction in the severity and improvement in the quality of life after using the drug. In a randomized, placebo-controlled, double-blind study by Thaçi et al., subjects were divided into six groups: five drug groups (having different dosing schedules) and one placebo group. The mean change in the Eczema Area and Severity Index (EASI) and Scoring Atopic Dermatitis (SCORAD) in the group receiving the dose of $300 \mathrm{mg} /$ week versus placebo at 16 weeks was found to be $-55.7 \%$ and $-43.1 \%$, respectively. Quality of life was assessed by the Dermatology Life Quality Index (DLQI) score. A mean change of $-61.6 \%$ as compared with the placebo was reported [23]. 
Beck et al. assessed the effects of dupilumab monotherapy as well as combination therapy (dupilumab with topical corticosteroids). The combination therapy was found to be most effective with $100 \%$ of patients achieving EASI-50 score (50\% reduction in EASI score), which was double as compared with the patients receiving topical corticosteroids with placebo $(\mathrm{p}=0.002)$. Pruritus scores reduced by $55.7 \%$ in the dupilumab group versus $15.1 \%$ in the placebo group $(\mathrm{p}<0.001)$ [24].

A phase 3 trial conducted by Blauvelt et al. for one year reported that patients receiving dupilumab with topical corticosteroids achieved co-primary endpoints of Investigator Global Assessment (IGA) 0/1 and EASI-75 (75\% reduction in EASI score) in a significantly higher number than the placebo groups $(\mathrm{p}<0 \cdot 0001)$. Quality of life improved by 10.5 on DLQI score in the group receiving $300 \mathrm{mg}$ weekly dose of the combination therapy (dupilumab plus topical corticosteroids) [25].

Guttman-Yassky et al. assessed skin biopsy specimens and blood samples from 54 patients who were randomly divided into the dupilumab and placebo groups. At 16 weeks, the lesional epidermal thickness was found to be significantly reduced in the dupilumab group compared with the placebo group $(\mathrm{p}=0.0002)$. The atopic dermatitis severity scores on EASI and SCORAD were also found to be notably reduced after the use of dupilumab [26].

Hamilton et al. performed transcriptome analyses of skin biopsy specimens from patients before and after treatment with dupilumab or placebo. It was found out that the expression of the genes normally involved in atopic dermatitis was reduced by $65 \%$ in the patients using $300 \mathrm{mg}$ weekly dose of dupilumab. The changes in clinical scores were also indicative of a reduction in disease severity upon using dupilumab. A change of 64.3\% was observed in the EASI score assessed after four weeks in the group using $300 \mathrm{mg}$ of dupilumab weekly [27].

In a double-blind, parallel-group dose-ranging study conducted in 2016 by Simpson et al., 380 patients were divided into six different groups (five drug groups with one placebo group). Patients using $300 \mathrm{mg}$ dupilumab weekly reported the most improvement in the symptoms of atopic dermatitis and the quality of life of patients. The Pruritus NRS (Numeric Rating Scale) score was significantly reduced ( $<<0.0001$ ) in most of the drug groups. There was a reduction of $75 \%$ in the anxiety score on Hospital Anxiety and Depression Scale (HADS) in patients using $300 \mathrm{mg}$ dupilumab weekly as compared with $22.2 \%$ improvement seen in the placebo group $(\mathrm{p}<0.05)$. The quality of life was also found to be significantly improved as evident from the DLQI and 5-dimension 3-level EuroQol (EQ-5D-3) scores $(\mathrm{p}<0.05)$ [28].

Table 2 summarizes the efficacy of dupilumab in terms of the commonly used scoring systems as reported in the studies mentioned. The table includes the results reported for the weekly dose of $300 \mathrm{mg}$ dupilumab with the exception of Guttman-Yassky et al. (200 mg weekly). 


\section{Cureus}

\begin{tabular}{|c|c|c|c|c|c|c|}
\hline \multirow[t]{2}{*}{ Authors } & \multirow[t]{2}{*}{ Year } & \multicolumn{5}{|l|}{ Efficacy } \\
\hline & & $\begin{array}{l}\text { Change in } \\
\text { SCORAD } \\
\text { (from } \\
\text { baseline) }\end{array}$ & Change in EASI score & $\begin{array}{l}\text { Change in Pruritus } \\
\text { NRS score (from } \\
\text { baseline) }\end{array}$ & $\begin{array}{l}\text { Change in } \\
\text { DLQI score } \\
\text { (from } \\
\text { baseline) }\end{array}$ & $\begin{array}{l}\text { Patients } \\
\text { achieving IGA } \\
\text { score } 0 \text { or } 1\end{array}$ \\
\hline $\begin{array}{l}\text { Thaçi et } \\
\text { al. [23] }\end{array}$ & 2016 & $-56 \cdot 9 \%$ & $-73.7 \%$ (from Baseline) & $-46.90 \%$ & $-59 \cdot 0 \%$ & $33 \%$ \\
\hline $\begin{array}{l}\text { Beck et } \\
\text { al. [24] }\end{array}$ & 2014 & & $\begin{array}{l}\text { Combination therapy EASI- } 50=100 \%(p<0.05) \\
\text { Dupilumab monotherapy EASI-50 }=85 \%(p<0.001) \\
\text { Combination therapy EASI-75 }=62 \% \text { Dupilumab } \\
\text { monotherapy EASI- } 75=62 \%\end{array}$ & $\begin{array}{l}\text { Dupilumab } \\
\text { monotherapy }= \\
-55.7 \% \text { Combination } \\
\text { therapy }=-70.7 \%(p \\
<0.05)\end{array}$ & & $\begin{array}{l}\text { Dupilumab } \\
\text { monotherapy = } \\
40 \%(p<0.001) \\
\text { Combination } \\
\text { therapy }=52 \%\end{array}$ \\
\hline $\begin{array}{l}\text { Blauvelt } \\
\text { et al. [25] }\end{array}$ & 2017 & $\begin{array}{l}\text { Combination } \\
\text { therapy }= \\
-63 \cdot 3 \%(p< \\
0 \cdot 0001)\end{array}$ & $\begin{array}{l}\text { Combination therapy EASI-75 }=64 \%(p<0.0001) \\
\text { and EASI- } 50=78 \%(p<0.0001)\end{array}$ & $\begin{array}{l}\text { Combination therapy } \\
=-4.1(p<0.0001)\end{array}$ & $\begin{array}{l}\text { Combination } \\
\text { therapy }=- \\
10.5(p< \\
0.0001)\end{array}$ & $\begin{array}{l}\text { Combination } \\
\text { therapy }=39 \%(p \\
<0.0001)\end{array}$ \\
\hline $\begin{array}{l}\text { Guttman- } \\
\text { Yassky } \\
\text { et al. [26] }\end{array}$ & 2019 & $\begin{array}{l}-54.8 \%(p< \\
0.0001)\end{array}$ & $\begin{array}{l}\text { EASI-75 }=66.7 \%(p=0.0001) \text { and EASI-50 }=77.8 \% \\
(p<0.0001)\end{array}$ & $-51.5 \%(p=0.0027)$ & & $\begin{array}{l}37.0 \%(p= \\
0.0006)\end{array}$ \\
\hline $\begin{array}{l}\text { Davis et } \\
\text { al. [29] }\end{array}$ & 2018 & & EASI-75 $=78.6 \%$ and EASI-50 $=92.9 \%$ & & & $50 \%$ \\
\hline $\begin{array}{l}\text { Hamilton } \\
\text { et al. [27] }\end{array}$ & 2014 & & EASI-50 $=71.4 \%$ & & & \\
\hline $\begin{array}{l}\text { Simpson } \\
\text { et al. [28] }\end{array}$ & 2016 & $\begin{array}{l}\text { SCORAD } \\
\text { sleep VAS }= \\
-4.3(p< \\
0.0001)\end{array}$ & & $-3.2(\mathrm{p}<0.0001)$ & $\begin{array}{l}-9.3(p< \\
0.0001)\end{array}$ & \\
\hline $\begin{array}{l}\text { Blauvelt } \\
\text { et al. [30] }\end{array}$ & 2019 & & EASI $-50=72.2 \%$ and EASI- $75=53.6 \%$ & -4.46 & & $44.30 \%$ \\
\hline
\end{tabular}

\section{TABLE 2: Efficacy of dupilumab}

Combination therapy refers to dupilumab plus topical corticosteroids.

SCORAD, Scoring Atopic Dermatitis; EASI, Eczema Area and Severity Index; Pruritus NRS, Pruritus Numeric Rating Scale; DLQI, Dermatology Life Quality Index; IGA, Investigator Global Assessment; EASI-50, 50\% reduction in EASI score; EASI-75, 75\% reduction in EASI score; SCORAD sleep VAS, Scoring Atopic Dermatitis sleep Visual Analogue Scale

\section{Safety and drug interactions}

Evidence from several trials suggests that treatment with dupilumab results in certain treatment-emergent adverse events. The most commonly reported adverse effects of dupilumab include headache, injection site reaction, conjunctivitis, and nasopharyngitis [23-26]. Herpes viral infections have also been reported by some researchers $[23,26]$.

Due to the potential effects of dupilumab on the cytochrome P450 (CYP450) enzyme system, FDA recommends close monitoring of patients taking concomitant CYP450 substrate drugs (such as warfarin and cyclosporine) [15]. In contrast, a study conducted by Davis et al. in 2018 failed to demonstrate any meaningful effect of dupilumab on the pharmacokinetics of CYP450 substrates [29].

A study by Blauvelt et al. concluded that dupilumab did not affect the responses to the non-live vaccines included in the trial [30]. The current recommendations by FDA caution only against the use of live vaccines in patients taking dupilumab [15].

\section{Other uses}

An increasing number of clinical trials suggest that the use of dupilumab reduces the severity of various allergic conditions due to its targeted action on specific cytokines. Corren et al. reported a significant 
improvement in the severity of asthma and the quality of life of the patients [31]. Other trials have similarly assessed the efficacy and safety of dupilumab in patients with uncontrolled asthma [32,33]. Similarly, the beneficial effects of dupilumab were reported in patients with conditions such as chronic rhinosinusitis and eosinophilic esophagitis [34,35].

\section{Conclusions}

Dupilumab is a recently approved drug for the management of atopic dermatitis, which works by inhibiting the signaling mechanisms involved in the pathogenesis of allergic skin conditions. The evidence from an increasing number of trials suggests that the benefits of dupilumab far outweigh its side-effects. It may be too early to declare dupilumab the ultimate drug of choice for atopic dermatitis, but the current evidence suggests that it has the potential to be the next wonder drug in dermatology.

\section{Additional Information}

\section{Disclosures}

Conflicts of interest: In compliance with the ICMJE uniform disclosure form, all authors declare the following: Payment/services info: All authors have declared that no financial support was received from any organization for the submitted work. Financial relationships: All authors have declared that they have no financial relationships at present or within the previous three years with any organizations that might have an interest in the submitted work. Other relationships: All authors have declared that there are no other relationships or activities that could appear to have influenced the submitted work.

\section{References}

1. Eichenfield LF, Tom WL, Chamlin SL, et al.: Guidelines of care for the management of atopic dermatitis: section 1. Diagnosis and assessment of atopic dermatitis. J Am Acad Dermatol. 2014, 70:338-51. 10.1016/j.jaad.2013.10.010

2. Silverberg JI, Hanifin JM: Adult eczema prevalence and associations with asthma and other health and demographic factors: a United States population-based study. J Allergy Clin Immunol. 2013, 132:1132-8. 10.1016/j.jaci.2013.08.031

3. Gittler JK, Shemer A, Suárez-Fariñas M, et al.: Progressive activation of $\mathrm{T}(\mathrm{H}) 2 / \mathrm{T}(\mathrm{H}) 22$ cytokines and selective epidermal proteins characterizes acute and chronic atopic dermatitis. J Allergy Clin Immunol. 2012, 130:1344-54. 10.1016/j.jaci.2012.07.012

4. De Benedetto A, Kubo A, Beck LA: Skin barrier disruption: a requirement for allergen sensitization? . J Invest Dermatol. 2012, 132:949-63. 10.1038/iid.2011.435

5. Ring J, Alomar A, Bieber T, et al.: Guidelines for treatment of atopic eczema (atopic dermatitis) part I . J Eur Acad Dermatol Venereol. 2012, 26:1045-60. 10.1111/j.1468-3083.2012.04635.x

6. Sidbury R, Tom WL, Bergman JN, et al.: Guidelines of care for the management of atopic dermatitis: Section 4. Prevention of disease flares and use of adjunctive therapies and approaches. J Am Acad Dermatol. 2014, 71:1218-33. 10.1016/j.jaad.2014.08.038

7. Sidbury R, Davis DM, Cohen DE, et al.: Guidelines of care for the management of atopic dermatitis: section 3. Management and treatment with phototherapy and systemic agents. J Am Acad Dermatol. 2014, 71:32749. 10.1016/j.jaad.2014.03.030

8. Gandhi NA, Bennett BL, Graham NM, Pirozzi G, Stahl N, Yancopoulos GD: Targeting key proximal drivers of type 2 inflammation in disease. Nat Rev Drug Discov. 2016, 15:35-50. 10.1038/nrd4624

9. Bao K, Reinhardt RL: The differential expression of interleukin-4 and interleukin-13 and its impact on type2 immunity. Cytokine. 2015, 75:25-37. 10.1016/j.cyto.2015.05.008

10. May RD, Fung M: Strategies targeting the interleukin-4/interleukin-13 axes in disease . Cytokine. 2015, 75:89-116. 10.1016/i.cyto.2015.05.018

11. Nomura I, Goleva E, Howell MD, et al.: Cytokine milieu of atopic dermatitis, as compared to psoriasis, skin prevents induction of innate immune response genes. J Immunol. 2003, 171:3262-9. 10.4049/jimmunol.171.6.3262

12. Zheng T, Oh MH, Oh SY, Schroeder JT, Glick AB, Zhu Z: Transgenic expression of interleukin-13 in the skin induces a pruritic dermatitis and skin remodeling. J Invest Dermatol. 2009, 129:742-51. 10.1038/jid.2008.295

13. Hussein YM, Ahmad AS, Ibrahem MM, Elsherbeny HM, Shalaby SM, El-Shal AS, Sabbah NA: Interleukin 13 receptors as biochemical markers in atopic patients. J Investig Allergol Clin Immunol. 2011, 21:101-7.

14. Sastre J, Dávila I: Dupilumab: a new paradigm for the treatment of allergic diseases . Investig Allergol Clin Immunol. 2018, 28:139-50. 10.18176/jiaci.0254

15. DUPIXENT (dupilumab) injection. (2017). Accessed: March 15, 2020: https://www.accessdata.fda.gov/drugsatfda_docs/label/2017/761055lbl.pdf.

16. Worm M, Simpson EL, Thaçi D, et al.: Efficacy and safety of multiple dupilumab dose regimens after initial successful treatment in patients with atopic dermatitis: a randomized clinical trial. JAMA Dermatol. 2019, 156:131-43. 10.1001/jamadermatol.2019.3617

17. Tofte SJ, Papp K, Sadick N, et al.: Efficacy and safety of dupilumab for the treatment of moderate-to-severe atopic dermatitis in adults: a pooled analysis of two phase 2 clinical trials. J Am Assoc Nurse Pract. 2018, 30:529-41. 10.1097/JXX.0000000000000088

18. Charman C, Williams H: Outcome measures of disease severity in atopic eczema . Arch Dermatol. 2000, 136:763-9. 10.1001/archderm.136.6.763

19. Chopra R, Silverberg JI: Assessing the severity of atopic dermatitis in clinical trials and practice . Clin Dermatol. 2018, 36:606-15. 10.1016/i.clindermatol.2018.05.012

20. Dermatology Life Quality Index. (2020). Accessed: March 15, 2020: 
https://www.cardiff.ac.uk/medicine/resources/quality-of-life-questionnaires/dermatology-life-qualityindex.

21. EQ-5D-3L. (2020). Accessed: March 15, 2020: https://euroqol.org/eq-5d-instruments/eq-5d-3l-about/.

22. Numerical Rating Scale (NRS). (2020). Accessed: March 15, 2020:

http://www.pruritussymposium.de/numericalratingscale.html.

23. Thaçi D, Simpson EL, Beck LA, et al.: Efficacy and safety of dupilumab in adults with moderate-to-severe atopic dermatitis inadequately controlled by topical treatments: a randomised, placebo-controlled, doseranging phase $2 \mathrm{~b}$ trial. Lancet. 2016, 387:40-52. 10.1016/S0140-6736(15)00388-8

24. Beck LA, Thaçi D, Hamilton JD, et al.: Dupilumab treatment in adults with moderate-to-severe atopic dermatitis. N Engl J Med. 2014, 371:130-9. 10.1056/NEJMoa1314768

25. Blauvelt A, de Bruin-Weller M, Gooderham M, et al.: Long-term management of moderate-to-severe atopic dermatitis with dupilumab and concomitant topical corticosteroids (LIBERTY AD CHRONOS): a 1-year, randomised, double-blinded, placebo-controlled, phase 3 trial. Lancet. 2017, 389:2287-303. 10.1016/S01406736(17)31191-1

26. Guttman-Yassky E, Bissonnette R, Ungar B, et al.: Dupilumab progressively improves systemic and cutaneous abnormalities in patients with atopic dermatitis. J Allergy Clin Immunol. 2019, 143:155-72. 10.1016/j.jaci.2018.08.022

27. Hamilton JD, Suárez-Fariñas M, Dhingra N, et al.: Dupilumab improves the molecular signature in skin of patients with moderate-to-severe atopic dermatitis. J Allergy Clin Immunol. 2014, 134:1293-300. 10.1016/j.jaci.2014.10.013

28. Simpson EL, Gadkari A, Worm M, et al.: Dupilumab therapy provides clinically meaningful improvement in patient-reported outcomes (PROs): a phase IIb, randomized, placebo-controlled, clinical trial in adult patients with moderate to severe atopic dermatitis (AD). J Am Acad Dermatol. 2016, 75:506-15. 10.1016/j.jaad.2016.04.054

29. Davis JD, Bansal A, Hassman D, et al.: Evaluation of potential disease-mediated drug-drug interaction in patients with moderate-to-severe atopic dermatitis receiving dupilumab. Clin Pharmacol Ther. 2018, 104:1146-54. 10.1002/cpt.1058

30. Blauvelt A, Simpson EL, Tyring SK, et al.: Dupilumab does not affect correlates of vaccine-induced immunity: a randomized, placebo-controlled trial in adults with moderate-to-severe atopic dermatitis. J Am Acad Dermatol. 2019, 80:158-67. 10.1016/j.jaad.2018.07.048

31. Corren J, Castro M, Chanez P, et al.: Dupilumab improves symptoms, quality of life, and productivity in uncontrolled persistent asthma. Ann Allergy Asthma Immunol. 2019, 122:41-9. 10.1016/j.anai.2018.08.005

32. Busse WW, Maspero JF, Rabe KF, et al.: Liberty Asthma QUEST: phase 3 randomized, double-blind, placebocontrolled, parallel-group study to evaluate dupilumab efficacy/safety in patients with uncontrolled, moderate-to-severe asthma. Adv Ther. 2018, 35:737-48. 10.1007/s12325-018-0702-4

33. Weinstein SF, Katial R, Jayawardena S, et al.: Efficacy and safety of dupilumab in perennial allergic rhinitis and comorbid asthma. J Allergy Clin Immunol. 2018, 142:171-7. 10.1016/j.jaci.2017.11.051

34. Bachert C, Han JK, Desrosiers M, et al.: Efficacy and safety of dupilumab in patients with severe chronic rhinosinusitis with nasal polyps (LIBERTY NP SINUS-24 and LIBERTY NP SINUS- 52): results from two multicentre, randomised, double-blind, placebo-controlled, parallel-group phase 3 trials. Lancet. 2019, 394:1638-50. 10.1016/S0140-6736(19)31881-1

35. Hirano I, Dellon ES, Hamilton JD, et al.: Efficacy of dupilumab in a phase 2 randomized trial of adults with active eosinophilic esophagitis. Gastroenterology. 2020, 158:111-22. 10.1053/j.gastro.2019.09.042 